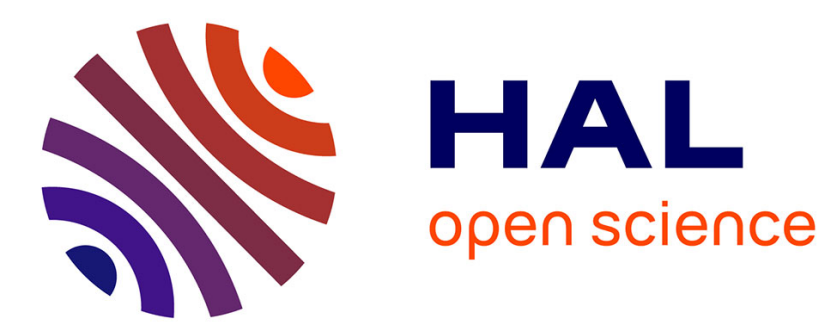

\title{
A new optical set-up for on-line following up the crystallization of polymers at high cooling rates
}

Simon Becker, Mathieu Brisebourg, Séverine A.E. Boyer

\section{To cite this version:}

Simon Becker, Mathieu Brisebourg, Séverine A.E. Boyer. A new optical set-up for on-line following up the crystallization of polymers at high cooling rates. 12th ESAFORM Conference on Material Forming, Apr 2009, Enschede, Netherlands. pp.Pages 853-856, 10.1007/s12289-009-0643-1 . hal-00509420

\section{HAL Id: hal-00509420}

https://hal-mines-paristech.archives-ouvertes.fr/hal-00509420

Submitted on 12 Aug 2010

HAL is a multi-disciplinary open access archive for the deposit and dissemination of scientific research documents, whether they are published or not. The documents may come from teaching and research institutions in France or abroad, or from public or private research centers.
L'archive ouverte pluridisciplinaire HAL, est destinée au dépôt et à la diffusion de documents scientifiques de niveau recherche, publiés ou non, émanant des établissements d'enseignement et de recherche français ou étrangers, des laboratoires publics ou privés. 


\title{
A NEW OPTICAL SET-UP FOR ON-LINE FOLLOWING UP THE CRYSTALLIZATION OF POLYMERS AT HIGH COOLING RATES
}

\author{
S. Becker ${ }^{1}$, M. Brisebourg, S.A.E. Boyer ${ }^{1 *}$ \\ ${ }^{1}$ MINES ParisTech, CEMEF - Centre de Mise en Forme des Matériaux, CNRS UMR 7635, 1 Rue \\ Claude Daunesse, 06904 Sophia Antipolis Cedex, France
}

\begin{abstract}
:
A new experimental procedure, named « Polymer High Cooling - Optics » and whose performance has been improved, is presented. It allows us to monitor on-line the quiescent crystallization of a polymer film under high and constant cooling rates. With such a set-up, two cooling control modes are available resulting into a range of relatively moderate (from 30 to $500{ }^{\circ} \mathrm{C} / \mathrm{min}$ ) and a range of high (from 500 to $1600^{\circ} \mathrm{C} / \mathrm{min}$ ) constant cooling rates. The crystallization experiments are observed by polarized light microscopy.

Preliminary results are presented. They are obtained on the $\alpha$-modification of one industrial grade of isotactic polypropylene, under relatively moderate constant cooling rates. Two relevant crystallization parameters are captured, the temperature of crystallization together with the spherulitic growth kinetics versus cooling rate. Accurate data are obtained. As well, they are in good agreement with comparable literature results compiled by Janeschitz-Kriegl [Macromolecules, 2006]. The obtained results seem to match the Hoffman and Lauritzen theory.

These discussions validate our « Polymer High Cooling - Optics » set-up in relatively moderate constant cooling rates. Next step is to demonstrate the suitability of this first generation set-up for higher constant cooling rates.
\end{abstract}

KEYWORDS: Industrial Processes, Liquid to Solid Transition, Isotactic Polypropylene, High Cooling Rates, One-line Optical Monitoring

\section{INTRODUCTION}

Among the large thermo-mechanical constraints that a polymer undergoes during industrial processes [1], high cooling rates are of prime importance. Consequently, the investigation of crystallization phenomena requires acquiring an expertise in constant high cooling rates, i.e., to develop well-controlled cooling procedures equivalent to those typically encountered in processing. This leads to propose new home-made devices and techniques as well as to interpret the new investigated data from existing models and/or from the formulation of new models.

In the present communication, the choice of an experimental framework and the design and performance of a new set-up are presented with the ability to well monitor on-line the crystallization of semi-crystalline polymers under constant and high cooling rates.

In a first part, a brief review of original conceptions employed to investigate polymer crystallization under high cooling rates is presented. The review is followed by the description of crystallization mechanism proposed by Hoffman and Lauritzen. In a second part, our own approach is presented. In a last part, results for relatively moderate cooling rates are discussed from a comparison with literature data and from a theoretical point of view.

\section{OVERVIEW ON GROWTH KINETICS TOGETHER WITH HIGH COOLING POLYMER CRYSTALLIZATION}

For on-line following the growth rate of spherulitic entities, hot stage microscopy is successfully used, but it requires original conceptions to reach high degrees of supercooling.

\subsection{OPTICAL ORIGINAL CONCEPTIONS}

Approaches dealing with the crystallization under constant and high cooling rates consist in designing and setting up model experiments. Ding \& Spruiell [2] developed a technique based on the earlier work of Magill [3] who introduced the light-depolarizing microscopy (LDM) technique as a method of investigating the overall kinetics of the crystallization process. Their technique allows studies of polymer films,

\footnotetext{
* Corresponding author: MINES ParisTech, Tel: 3349395 75 65, Fax: 33492389752

Email address: Severine.BOYER@mines-paristech.fr
} 
$150 \mu \mathrm{m}$ in thickness, under non-isothermal conditions up to $5000^{\circ} \mathrm{C} / \mathrm{min}$. Brucato, Piccarolo and Titomanlio supplied a general experimental route for which thin polymeric samples, 50-100 $\mu \mathrm{m}$ in thickness, are quenched at very high cooling rates up to $2000^{\circ} \mathrm{C} / \mathrm{s}$ by rapidly moving the polymer sample from a hot into a cold section. The experimental procedure involves the recording of the thermal history that the polymer undergoes during crystallization [4-6]. Moreover, it involves simultaneous detection of overall and depolarized light intensities emerging in the course of polymer crystallization [7].

\subsection{HOFFMAN AND LAURITZEN MODEL}

Hoffman \& Lauritzen proposed and revisited repeatedly a polymer crystal growth mechanism or secondary nucleation kinetic theory [8,9]. They classify different regimes of lamellar growth: regime I, regime II and regime III, respectively. These regime differ by the deposit mechanism of molecular nuclei on the growing lamella or, in other words, by the relative values of the nucleation rate on one substrate and of the substrate completion rate. The regime $\mathrm{I}$ is observed for high crystallization temperature where one surface nucleus causes the completion of the entire substrate; the regime II is observed when multiple surface nuclei begin to occur on the substrate because of a larger undercooling; the regime III is similar to the regime II but is entered when distance separation between characteristics of the substrate in the Regime II approaches the value of a molecular stem. In the context of Hoffman \& Lauritzen secondary nucleation theory, the linear growth rate $G$ of a crystalline entity (e.g., spherulite) is dependent on the degree of undercooling $\Delta T$ as presented in Equation (1) below.

$$
G=G_{0} \exp \left(-\frac{U^{*}}{R\left(T_{c}-T_{\infty}\right)}-\frac{K_{g}}{T_{c}(\Delta T) f}\right)
$$

$G_{o}$ is a pre-exponential term which is not strongly dependent on temperature. $U^{*}$ and $T_{\infty}$ are the VogelFulcher-Tamman-Hesse (VFTH) parameters. $U^{*}$ is the activation energy for the transportation of segments of molecules across the melt/solid surface boundary and is usually given by a universal value of $1500 \mathrm{cal} / \mathrm{mol}$. $T_{\infty}$ is the temperature where the molecular motion ceases, with $T_{\infty}=T_{g}-30 . T_{c}$ is the crystallization temperature. $R$ is the universal gas constant. $\Delta T$ is the degree of undercooling with $\Delta T=T_{m}^{0}-T_{c} \cdot f$ is a factor used to correct the temperature dependence of the heat of fusion (i.e., $f=2 T_{c} /\left(T_{c}+T_{m}^{0}\right)$ ). $K_{g}$ is the nucleation parameter defined by Equation (2). $\xi$ equals 2 for the regime II and 4 for the regime I and III, $b_{0}$ denotes the crystal layer thickness along the growth direction, $\sigma$ and $\sigma_{e}$ are the lateral and fold surface free energy, respectively. $T_{m}^{0}$ is the equilibrium melting temperature. $k$ is the Boltzmann constant. $\Delta H_{f}^{0}$ is the equilibrium heat of fusion.

$$
K_{g}=\frac{\xi b_{0} \sigma \sigma_{e} T_{m}^{0}}{k \Delta H_{f}^{0}}
$$

Referring to Equation (1), the first exponential term, $\exp \left(-U^{*} / R\left(T_{c}-T_{\infty}\right)\right)$, corresponds to the diffusion of polymer molecules or segments of them from the equilibrium melt onto the growth surface. The second exponential term, $\exp \left(-K_{g} / T_{c}(\Delta T) f\right)$, relates to the formation of the critical nucleus on the growth surface. Intuitively, from the competing contributions of the transport and nucleation terms, one expects that there should be a maximum in the growth rate data at a temperature between the glass transition and the melting temperature, when plotted as a function of $T_{c}$.

Equation (1) can be rewritten into Equation (3) as given here after.

$$
\ln G+\frac{U^{*}}{R\left(T_{c}-T_{\infty}\right)}=\ln G_{0}-\frac{K_{g}}{T_{c}(\Delta T) f}
$$

It is possible to factor out the contribution of the transport term to the growth rate through the plotting $\ln G+U^{*} / R\left(T_{c}-T_{\infty}\right)$ versus $1 / T_{c}(\Delta T) f$. Nucleation parameter $K_{g}$ can be then deduced.

\section{DESIGN AND INSTRUMENTATION}

This part describes both the material used and the newly revisited home-made technique named «Polymer High Cooling - Optics ».

\subsection{MATERIALS}

One isotactic polypropylene (iPP) grade for injection molding is used. The commercial reference is $3250 \mathrm{MR} 1$ (ATOFINA Company, France) (now named PPH9081, TOTAL PETROCHEMICALS) with a weight average molecular weight $M_{w}$ of $213000 \mathrm{~g} / \mathrm{mol}$ and a polydispersity $P D$ of 5.

\subsection{NEW 'POLYMER HIGH COOLING' SET-UP}

\subsubsection{Polarizing Optical Microscopy}

Optical light microscopy from a LEICA-DMRX microscope (Leica-Microsystèmes, France) with a CMOS camera is used to one-line monitor the structural evolution. Optical micrographs are taken between crossed polarizers, $+/-90^{\circ}$ rotated.

\subsubsection{Constant High Cooling rates control}

A specially designed hot-stage, optically transparent, is used. It is equipped with a flux control system that can heat or cool a gas passing over the sample. The gaseous flux is used as a heating and cooling transfer medium. 
The sample is in a shape of film and has a thickness of about $50 \mu \mathrm{m}$ in the area of observation. The sample temperature is continuously measured by a $\mathrm{K}$ type thermocouple of $0.25 \mathrm{~mm}$ in diameter embedded directly in the polymer sample. This eliminates lag time between the sample and the temperature of the surroundings, i.e., air/polymer heat transfer. To optimize the on-line time/polymer temperature following, attention was paid to locate the thermocouple, in contact with the polymer, very near to the observation area. The polymer plus thermocouple set was sandwiched by a pair of clean thin glass slides.

The polymer is heated and cooled down by the flowing gaseous heat transfer medium. The sample is heated and cooled down in the same location in the hot-stage chamber, i.e., without any transfer.

The heating of the sample was accomplished by passing air through a separate heating unit or throttle chamber. The heat exchanger chamber receives the gaseous flux coming either from the cooling line or from the heating line. When the cooling process begins, the hot air is mixed with cold air. The rate of mixing is done by controlling proportionally the gap of the two lines.

A home-made program was especially written under Labview control. It allows us to simultaneously program the cooling rates, the temperature profile of the sample and the monitoring of optical observation. The program proposes two cooling modes: a proportional one using a rotating throttle to control constant cooling rates at relatively moderate values, and an 'all-or-none' one using an 'open-close' valve for cold flux to attain high cooling rates.

The experiments were performed with the same polymer film. The protocol is: prior to crystallization, the sample is kept in the molten state at about $215^{\circ} \mathrm{C}$ for about $90 \mathrm{~s}$ to erase the thermal history and to avoid its degradation, enabling thus the processing of the sample for many cycles. Then the experiments in the constant nonisothermal mode consisted of cooling scans between 215 and $50^{\circ} \mathrm{C}$.

The actual operating range of relatively moderate and constant cooling rates is $30-500 \circ \mathrm{C} / \mathrm{min}$. The temperature profiles programmed and measured were accordingly as shown in Figure 1. It proves that the sample temperature is well-controlled.

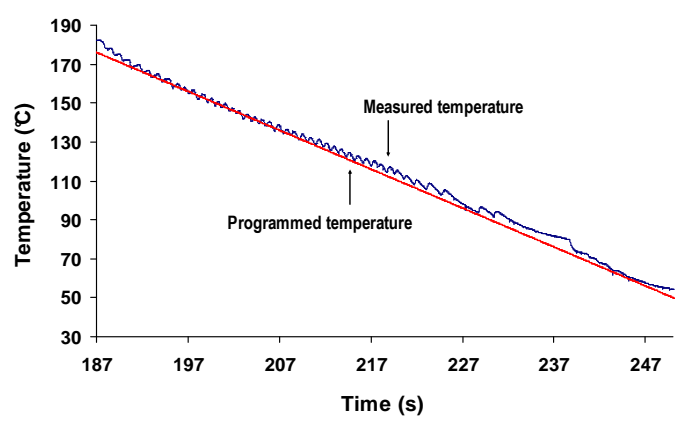

Figure 1: Temperature-time evolution of iPP sample at a constant cooling rate of $144^{\circ} \mathrm{C} / \mathrm{min}$.

\section{RESULTS \& DISCUSSION}

The growth kinetics resulting from relatively moderate and constant cooling rates are presented together with the understanding of crystallization mechanism by means of the theory of Hoffman and Lauritzen.

\subsection{CRYSTAL GROWTH UNDER RELATIVELY MODERATE CONSTANT COOLING RATES}

Figure 2 shows an illustration of the optical snapshots indicating the radial development of crystalline entities under constant cooling rates.
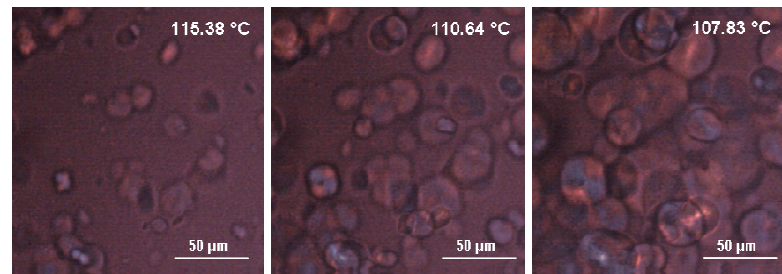

Figure 2: Development of crystalline iPP spherulites at a constant cooling rate of $144{ }^{\circ} \mathrm{C} / \mathrm{min}$.

Figure 3 represents the relationship between the growth rates of iPP $\alpha$-spherulites in the quiescent melt and $T_{c}$ resulting from our new investigation. Additionally, the results of many authors, compiled by Janeschitz-Kriegl [10], are visible.

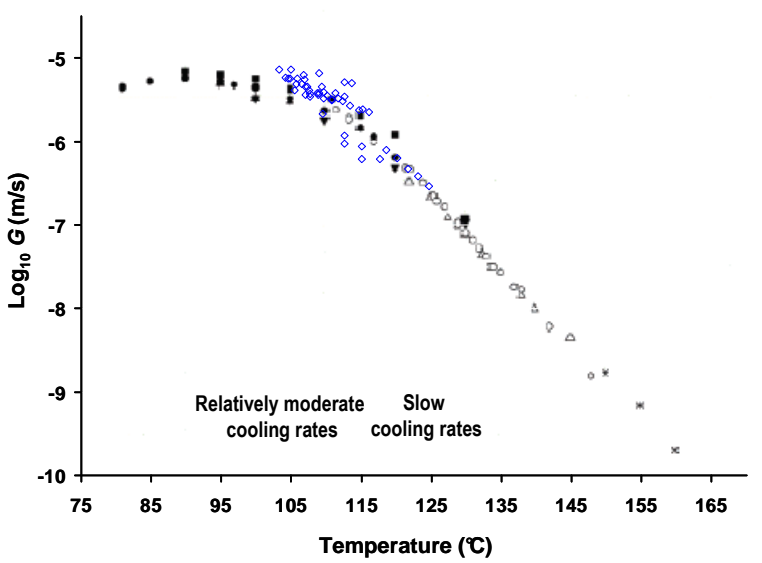

Figure 3: Compilation of iPP $\alpha$-spherulites growth rates versus the crystallization temperatures. Blue open diamonds are from the present investigation at relatively moderate cooling rates (from 50 to $500{ }^{\circ} \mathrm{C} / \mathrm{min}$ ); data at slow cooling rates are from an investigation using a conventional Mettler hot-stage (at 3 and $10^{\circ} \mathrm{C} / \mathrm{min}$ ). Open symbols are from Lovinger et al., 1977 (rhombs), Padden \& Keith, 1959 (circles), Von Falkai, 1960 (triangles), Olley \& Bassett, 1989 (stars). Closed symbols are from: $\beta$-transcrystallization, Ratajski, 1993 (closed squares), microtome slices, Ratajski \& Janeschitz-Kriegl, 1996 (closed circles), DSC, Paulik, 1993 (closed triangles pointed upward) and Ratajski \& Janeschitz-Kriegl, 1996 (closed diamonds), incubation times, Ratajski \& Janeschitz-Kriegl, 1996 (closed triangles pointed downward). Literature data are from reference [10]. 
Our results are in good agreement with the comparable ones from the literature. It is evident that, in the high temperature region, i.e., before attaining the maximum at about $T_{c}=90^{\circ} \mathrm{C}$, the spherulitic growth rate follows the typical bell-shaped dependence with $T_{c}$.

At the end of the study, the spherulitic phase of the investigated iPP sample was verified by X-Ray analysis. The condition pertains to crystallization of the $\alpha$-form.

\subsection{REGIME OF CRYSTALLIZATION}

To study the regime of crystallization according to the Hoffman and Lauritzen theory, Equation (3) is graphically represented in Figure 4.

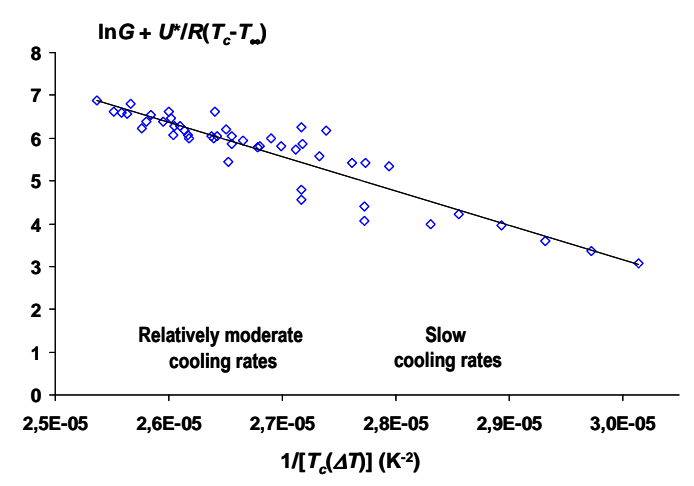

Figure 4: Analysis of the spherulitic growth rates of iPP versus the crystallization temperatures $T_{c}$ based on the Lauritzen \& Hoffman secondary nucleation theory with $T_{m}^{0}=208{ }^{\circ} \mathrm{C}$ and $T_{g}=-21^{\circ} \mathrm{C}$.

The regime III is observed when crystallization occurs at relatively moderate cooling rates. The nucleation exponent $K_{g}$ was estimated to $8.0110^{5} \mathrm{~K}^{2}$. The value is in good agreement with literature data on iPP [11].

\section{CONCLUSIONS}

The design and performances of a newly revisited homemade optical experimental set-up to monitor on-line structure development with a proper control of high and constant cooling rates are described. Spherulitic growthrate was measured at relatively moderate (from 30 to 500 ${ }^{\circ} \mathrm{C} / \mathrm{min}$ ) constant cooling rates. Results obtained match the data base done by Janeschitz-Kriegl as well as the Hoffman and Lauritzen theory.

Before going to implement such information into new crystallization models and then into numerical simulation, the effect of higher constant cooling rates (from 500 to $1600{ }^{\circ} \mathrm{C} / \mathrm{min}$ ) on crystallization event needs further investigations. Moreover, the reliability of the growth kinetics data will be enhanced by comparison with the results of alternative methods, i.e., overall kinetics from constant high cooling rate differential scanning calorimetry.

\section{ACKNOWLEDGEMENT}

The authors present their acknowledgements to Alain le Floc'h, Francis Fournier, Thierry Collin, and Lionel Freire for the help during the revisit of the set-up.

\section{REFERENCES}

[1] S.A.E. Boyer, L. Silva, M. Gicquel, S. Devisme, J.L. Chenot, and J.-M. Haudin. How to determine the parameters of polymer crystallization for modelling the injection-molding process ? In 11th ESAFORM Conference on Material Forming, Lyon (France), 2008.

[2] Z. Ding, J.E. Spruiell. An experimental method for studying nonisothermal crystallization of polymers at very high cooling rates. J. Polym. Sci. B. Polym. Phys., 34:2783-2804, 1996.

[3] J.H. Magill. A new technique for following rapid rates of crystallization II isotactic polypropylene. Polymer, 3:35-42, 1962.

[4] S. Piccarolo, G. Saiu, V. Brucato, G. Titomanlio. Crystallization of polymer melts under fast cooling. II High-purity iPP. J. Appl. Polym. Sci., 46:625-634, 1992.

[5] V. Brucato, S. Piccarolo, V. La Carruba. An experimental methodology to study polymer crystallization under processing conditions. The influence of high cooling rates. Chem. Eng. Sci., 57:4129-4143, 2002.

[6] I. Cocorullo, R. Pantani, G. Titomanlio. Crystallization kinetics and solidified structure in iPP under high cooling rates. Polymer, 44: 307-318, 2003.

[7] G. Lamberti, F. De Santis, V. Brucato, G. Titomanlio. Modeling the interactions between light and crystallization polymer during fast cooling. Appl. Phys. A, 78:895-901, 2004.

[8] J.I. Lauritzen Jr., J.D. Hoffman. Theory of formation of polymer crystals with folded chains in dilute solution. J. Res. Natl. Bur. Stand. A, 64:73$102,1960$.

[9] J.D. Hoffman, R.L. Miller. Kinetics of crystallization from the melt and chain folding in polyethylene fractions revisited: theory and experiment. Polymer, 38:3151-3212, 1997.

[10] H. Janeschitz-Kriegl. Phases of flow-induced crystal-lization of i-PP: how remote pieces of the puzzle appear to fit. Macromolecules, 39:44484454, 2006.

[11] J. Xu, S. Srinivas, H. Marand, P. Agarwal. Equilibrium melting and undercooling dependence of the spherulitic growth rate of isotactic polypropylene. Macromolecules, 31:8230-8242, 1998. 